\title{
$A b$ initio nuclear response functions for dark matter searches
}

\author{
D. Gazda, R. Catena, and C. Forssén \\ Department of Physics, Chalmers University of Technology, SE-412 96 Göteborg, Sweden
}

(Received 1 February 2017; published 30 May 2017)

\begin{abstract}
We study the process of dark matter particles scattering off ${ }^{3,4} \mathrm{He}$ with nuclear wave functions computed using an $a b$ initio many-body framework. We employ realistic nuclear interactions derived from chiral effective field theory at next-to-next-to-leading order (NNLO) and develop an ab initio scheme to compute a general set of different nuclear response functions. In particular, we then perform an accompanying uncertainty quantification on these quantities and study error propagation to physical observables. We find a rich structure of allowed nuclear responses with significant uncertainties for certain spin-dependent interactions. The approach and results that are presented here establish a new framework for nuclear structure calculations and uncertainty quantification in the context of direct and (certain) indirect searches for dark matter.
\end{abstract}

DOI: 10.1103/PhysRevD.95.103011

\section{INTRODUCTION}

Convincing evidence for the presence of dark matter in the Universe has been gathered over the past decades [1]. In the standard paradigm of modern cosmology, dark matter is a weakly interacting massive particle (WIMP) [2-4]. On cosmological scales, it behaves like a dissipationless and nonrelativistic fluid from the beginning of cosmological structure formation until the present time [5]. The detection of dark matter particles in a laboratory or in space is currently a priority of astroparticle physics. The experimental technique known as direct detection is expected to play a key role in this context [6]. It searches for nuclear recoil events induced by the nonrelativistic scattering of Milky Way dark matter particles in low-background detectors. Reliable nuclear physics input is therefore needed for the interpretation of data from such experiments. However, there is currently a gap between the treatment of the nuclear physics input in the field of dark matter studies and the level of sophistication that has been reached in modern theoretical nuclear physics. It is the main purpose of this work to fill this gap and introduce a more systematic approach, with a solid theoretical underpinning, that also allows us to explore and quantify various sources of uncertainties. The frameworks that will be used to achieve this goal are effective field theories (EFTs) and nuclear ab initio manybody methods.

Effective theory methods have already proven to be a very powerful tool in the analysis of dark matter detection experiments [7-9]. The main advantage of the effective theory approach to dark matter is that it allows for a modelindependent analysis of available data. In contrast, signs of important physical properties can be obscured when using a simplistic model for dark matter interactions. At the same time spurious correlations among physical observables can be enforced through an inappropriately small number of model parameters. Two main approaches have been used when constructing an effective theory for WIMP-nucleus scattering. In both cases the end result is an EFT with nonrelativistic nucleon and WIMP fields as the relevant degrees of freedom. Firstly, one can consider a specific set of effective interaction terms at the quark level defined at the hadronic scale and use chiral symmetry constraints to estimate the hierarchy among one- and two-nucleon currents [10-15]. This approach is very appealing since similar constraints are used in the construction of nuclear forces [16-18]. However, the matching of standard model fields to hadronic-level operators is an intricate problem; see e.g. Ref. [19] with lattice QCD results and a brief discussion of potential power-counting issues that are relevant for mesonexchange current contributions to the WIMP-nucleus interaction. The chiral EFT approach also allows, in principle, a mapping to the parameter space of new-physics models [15]. Such a mapping is not straightforward and requires us to take into account the evolution of the WIMP effective operators from the mediator mass scale to the lowenergy hadronic scale that is probed by direct detection experiments [20].

Alternatively, one can integrate out the QCD dynamics and construct directly an EFT in which nonrelativistic nucleon and WIMP fields are the degrees of freedom [9]. This so-called NREFT generates the most general set of WIMP-nucleon interactions based only on the requirement of Galilean invariance and momentum conservation. In this approach the connection to symmetries of QCD is lost, but can be recovered by matching to the chiral EFT framework discussed above. In this context we mention explicitly the work by Hoferichter et al. [14] to present a common chiral power-counting scheme and to match it to the NREFT operator basis. Most importantly, however, the NREFT framework is less restrictive with respect to the assumptions on the underlying quark-level dark matter interactions and the type of dark matter particle. There are possible dark matter-quark interactions, such as e.g. a dimension-6 
tensor-tensor type [21], that have not yet been analyzed in the chiral EFT framework and therefore may alter some of its conclusions. At the moment there is no experimental evidence that would favor any particular form of the underlying dark-matter particle interactions and we will therefore work within the general NREFT framework. We stress, however, that the $a b$ initio nuclear-physics method that we present here can be applied to both EFT approaches as its starting point is the interaction at the nucleon level.

The construction of the dark matter-nucleus interaction is the next step in the effective-theory approach to dark matter detection. Following Fitzpatrick et al. [9], eight nuclear response functions can be generated in the dark matter elastic scattering by nuclei. The interpretation of any dark matter experiment probing the dark matter-nucleus interaction is unavoidably affected by the uncertainties within which these nuclear response functions are known. In the simplest treatment, only spin-independent interactions are considered and phenomenological nuclear response functions-so-called "Helm form factors"- are used. More recently, many additional responses have been considered and more sophisticated nuclear-structure calculations have been performed using the shell model (SM) [11-13,22-25]. The SM is arguably a very successful phenomenological model for nuclear structure; see e.g. Refs. [26,27] for general overviews. Its configuration space comprises a relatively small number of "active" particles outside a core of nucleons that are frozen in the lowestenergy orbitals and not included in the calculation. This significant truncation of the model space is often critical for allowing any kind of solution to the many-body problem. The residual valence-space interaction should, in principle, incorporate effects from degrees of freedom that are not explicitly included in the model space. In practice, its construction typically corresponds to the introduction of free fitting parameters that are tuned so that the model reproduces (with an acceptable accuracy) energy spectra and/or other observables in the region of interest. The ability to quantify theoretical uncertainties associated with predicted nuclear matrix elements, and consequently in the constructed form factors, becomes severely restricted in such an approach. Only very recently it was shown how to obtain residual effective valence-space interactions starting from the underlying microscopic internucleon interaction in a systematic, nonperturbative framework using $a b$ initio methods [28-30]. However, it remains to be studied how theoretical model uncertainties can be quantified.

In recent years, $a b$ initio methods [31-36] have matured to a level where precise nuclear many-body calculations can be performed starting from nucleons as the relevant degrees of freedom and using realistic internucleon interactions. Furthermore, the use of EFTs for the description of these nuclear interactions provides a systematic approach that offers an estimate of the inherent model error. Significant progress in the quantification of truncation errors in EFT was reported recently [37-39] and also employed to provide theoretical uncertainties in nuclear structure calculations $[39,40]$ by combining ab initio manybody methods and chiral EFT interactions. It is a specific aim of this work to demonstrate how nuclear uncertainties can be quantified, at least for selected proof-of-principle cases, using $a b$ initio methods and realistic internucleon interactions.

Experiments whose analysis are affected by these uncertainties are dark matter direct detection experiments, with various detector materials, and neutrino telescopes searching for neutrinos from dark matter annihilations in the Sun and the Earth. In this work we concentrate on the former ones, and in particular on detector designs with sensitivities to the direction of nuclear recoils. Such designs are currently in a research and development stage. They are of particular interest for the present analysis in that helium, and especially ${ }^{3} \mathrm{He}$, is one of the target materials explored in this context [41,42]. For such a light target nucleus, ab initio nuclear structure calculations are straightforward, which allows a more robust uncertainty quantification. Furthermore, the use of ${ }^{3} \mathrm{He}$ for dark matter detection is interesting for other reasons [43-45]: it is an ideal target for the detection of light dark matter particles; neutron rejection can easily be achieved through the process $n+{ }^{3} \mathrm{He} \rightarrow p+{ }^{3} \mathrm{H}+764 \mathrm{keV}$; it has no intrinsic $\mathrm{x}$-ray emission and a low natural radioactive background; it can be polarized; and it allows us to probe the spin of the dark matter particle. As far as ${ }^{4} \mathrm{He}$ is concerned, its use for dark matter detection has recently been considered in [46]. In this investigation we will focus on hypothetical ${ }^{3} \mathrm{He}$ and ${ }^{4} \mathrm{He}$ detectors with directional sensitivity.

The article is organized as follows. In Sec. II we first review the nonrelativistic effective theory of dark matternucleon interactions (Sec. II A) and then introduce the $a b$ initio no-core shell model technique for the calculation of nuclear matrix elements in a Jacobi, relative-coordinate basis (Sec. II B). The nuclear many-body problem is solved with chiral nuclear interactions as input and these will be introduced in Sec. II C. Results are presented in Sec. III, focusing on nuclear response functions in Sec. III A and on rates of dark matter-nucleus scattering events at directional detectors in Sec. III B. We conclude with an outlook in Sec. IV.

\section{METHODOLOGY}

\section{A. Dark matter-nucleon and nucleus interaction}

Consider the nonrelativistic scattering of a dark matter particle $\chi$ by a single nucleon $N: \chi(\mathbf{k})+N(\mathbf{p}) \rightarrow$ $\chi\left(\mathbf{k}^{\prime}\right)+N\left(\mathbf{p}^{\prime}\right)$, where initial and final three-dimensional momenta are denoted by $\mathbf{k}$ and $\mathbf{p}$, and $\mathbf{k}^{\prime}$ and $\mathbf{p}^{\prime}$, respectively. Three-dimensional momentum conservation and Galilean invariance, i.e. the invariance under constant shifts of particle velocities, constrain the transition 
amplitude, $\mathcal{M}$, for this process. Momentum conservation implies that only three of the four momenta $\mathbf{k}, \mathbf{p}, \mathbf{k}^{\prime}$ and $\mathbf{p}^{\prime}$ are independent. The momentum transfer $\mathbf{q}=\mathbf{k}-\mathbf{k}^{\prime}, \mathbf{k}$ and $\mathbf{p}$ form a possible set of independent momenta. Galilean invariance implies that $\mathcal{M}$ cannot depend on $\mathbf{k}$ and $\mathbf{p}$ separately, but only on a Galilean invariant combination of them, for instance $\mathbf{v}=\mathbf{k} / m_{\chi}-\mathbf{p} / m_{N}$, where $m_{\chi}$ and $m_{N}$ are the dark matter particle and nucleon mass, respectively. Here $\mathbf{v}$ is the dark matter-nucleon relative velocity, and $\mathbf{q}$ is per se Galilean invariant. We conclude that in general, $\mathcal{M}=\mathcal{M}\left(\mathbf{q}, \mathbf{v}, \mathbf{S}_{\chi}, \mathbf{S}_{\mathbf{N}}\right)$, where $\mathbf{S}_{\chi}$ and $\mathbf{S}_{\mathbf{N}}$ are the dark matter and nucleon spin, respectively.

Next, we focus on the nonrelativistic quantum mechanical Hamiltonian $\hat{\mathcal{H}}_{\chi N}$ underlying the scattering amplitude $\mathcal{M}$. At the quantum mechanical level, any interaction operator describing the nonrelativistic limit of dark matternucleon interactions can be expressed in terms of four Hermitian operators [9]: $i \hat{\mathbf{q}}$, where $\hat{\mathbf{q}}$ is the momentum transfer operator; the transverse relative velocity operator, $\hat{\mathbf{v}}^{\perp}$; and the dark matter particle and nucleon spin operators, $\hat{\mathbf{S}}_{\chi}$ and $\hat{\mathbf{S}}_{N}$, respectively. By construction $\hat{\mathbf{v}}^{\perp} \cdot \hat{\mathbf{q}}=0$. Without further restrictions, $\hat{\mathcal{H}}_{\chi N}$ can in principle include an infinite number of interaction operators: all scalar combinations of $i \hat{\mathbf{q}}, \hat{\mathbf{v}}^{\perp}, \hat{\mathbf{S}_{\chi}}$ and $\hat{\mathbf{S}_{N}}$. However, when $|\mathbf{q}|$ is small compared to the mass of the particle that mediates the dark matter-nucleon interaction, $\hat{\mathcal{H}}_{\gamma N}$ can be expanded in powers of $\hat{\mathbf{q}}$. Truncating the expansion at second order, only 14 independent Galilean invariant interaction operators arise if dark matter has spin less than or equal to $1 / 2$ [24]. For spin 1 dark matter, two additional operators can be constructed [47], although these are only relevant when specific operator interference patterns are not negligible. We list the interaction operators $\hat{\mathcal{O}}_{j}$ considered in this study in Table I, using the notation introduced in [24] and an index $j$ to label them.

TABLE I. Interaction operators defining the effective theory of dark matter-nucleon interactions. The operator $\mathbb{1}_{\chi N}$ is the identity in the two-particle spin space. Here $m_{N}$ is the nucleon mass and all interaction operators have the same mass dimension. For simplicity, we omit the nucleon index $i$.

\begin{tabular}{|c|c|}
\hline $\begin{array}{l}\hat{\mathcal{O}}_{1}=\mathbb{1}_{\chi N} \\
\hat{\mathcal{O}}_{3}=i \hat{\mathbf{S}}_{N} \cdot\left(\frac{\hat{\mathbf{q}}}{m_{N}} \times \hat{\mathbf{v}}^{\perp}\right) \\
\hat{\mathcal{O}}_{4}=\hat{\mathbf{S}}_{\chi} \cdot \hat{\mathbf{S}}_{N} \\
\hat{\mathcal{O}}_{5}=i \hat{\mathbf{S}}_{\chi} \cdot\left(\frac{\hat{\mathbf{q}}}{m_{N}} \times \hat{\mathbf{v}}^{\perp}\right) \\
\hat{\mathcal{O}}_{6}=\left(\hat{\mathbf{S}}_{\chi} \cdot \hat{\mathbf{q}}\right)\left(\hat{\mathbf{S}}_{N} \cdot \frac{\hat{\mathbf{q}}}{m_{N}}\right) \\
\hat{\mathcal{O}}_{7}=\hat{\mathbf{S}}_{N} \cdot \hat{\mathbf{v}}^{\perp} \\
\hat{\mathcal{O}}_{8}=\hat{\mathbf{S}}_{\chi} \cdot \hat{\mathbf{v}}^{\perp}\end{array}$ & 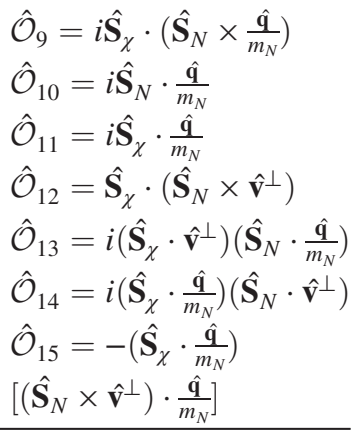 \\
\hline
\end{tabular}

The operators in Table I define a nonrelativistic theory called effective theory of dark matter-nucleon interactions (NREFT). Initially formulated in [7-9,24,48], it has later been developed in [10-12,21,47,49-64]. In this context, the most general Hamiltonian density for nonrelativistic dark matter-nucleus interactions is

$$
\hat{\mathcal{H}}_{\chi A}=\sum_{i=1}^{A} \sum_{\tau=0,1} \sum_{j} c_{j}^{\tau} \hat{\mathcal{O}}_{j}^{(i)} t_{(i)}^{\tau},
$$

where $A$ is the mass number of the target nucleus. The matrices $t_{(i)}^{0}=\mathbb{1}_{2 \times 2}$ and $t_{(i)}^{1}=\tau_{3}$, where $\tau_{3}$ is the third Pauli matrix, are defined in the isospin space of the $i$ th nucleon. Isoscalar and isovector coupling constants are denoted by $c_{j}^{0}$ and $c_{j}^{1}$, respectively. They are linearly related to the coupling constants for protons and neutrons ${ }^{1}$ : $c_{j}^{p}=\left(c_{j}^{0}+c_{j}^{1}\right), \quad c_{j}^{n}=\left(c_{j}^{0}-c_{j}^{1}\right)$, and have dimension $[\text { mass }]^{-2}$. Equation (1) is valid under the assumption that the dark matter-nucleus interaction is the sum of dark matter interactions with the individual nucleons. Corrections beyond this (impulse) approximation are discussed in [10-12,14,15,65].

We derive the differential cross section for dark matternucleus scattering from the Hamiltonian density in Eq. (1):

$$
\begin{aligned}
\frac{\mathrm{d} \sigma}{\mathrm{d} q^{2}}= & \frac{1}{(2 J+1) v^{2}} \sum_{\tau, \tau^{\prime}}\left[\sum_{\ell=M, \Sigma^{\prime}, \Sigma^{\prime \prime}} R_{\ell}^{\tau \tau^{\prime}}\left(v_{T}^{\perp 2}, \frac{q^{2}}{m_{N}^{2}}\right) W_{\ell}^{\tau \tau^{\prime}}\left(q^{2}\right)\right. \\
& \left.+\frac{q^{2}}{m_{N}^{2}} \sum_{m=\Phi^{\prime \prime}, \Phi^{\prime \prime} M, \tilde{\Phi}^{\prime}, \Delta, \Delta \Sigma^{\prime}} R_{m}^{\tau \tau^{\prime}}\left(v_{T}^{\perp 2}, \frac{q^{2}}{m_{N}^{2}}\right) W_{m}^{\tau \tau^{\prime}}\left(q^{2}\right)\right],
\end{aligned}
$$

where $J$ is the target nucleus spin, $v$ is from now onwards the dark matter-nucleus relative velocity, and $v_{q}^{\perp 2}=$ $v^{2}-q^{2} /\left(4 \mu_{\chi A}^{2}\right)$. Here $q \equiv|\mathbf{q}|$ and $\mu_{\chi A}$ is the dark matternucleus reduced mass. The eight dark matter response functions $R_{\ell}^{\tau \tau^{\prime}}$ and $R_{m}^{\tau \tau^{\prime}}$ depend on the isoscalar and isovector coupling constants $c_{j}^{\tau}, q^{2} / m_{N}^{2}$ and $v_{q}^{\perp 2}$. They were first derived in $[9,24]$ and are listed in the Appendix.

The eight nuclear response functions $W_{\ell}^{\tau \tau^{\prime}}$ and $W_{m}^{\tau \tau^{\prime}}$ in Eq. (2) are given by

$$
\begin{aligned}
W_{A B}^{\tau \tau^{\prime}}\left(q^{2}\right)= & \sum_{L \leq 2 J}\left\langle J, T, M_{T}\left\|\hat{A}_{L ; \tau}(q)\right\| J, T, M_{T}\right\rangle \\
& \times\left\langle J, T, M_{T}\left\|\hat{B}_{L ; \tau^{\prime}}(q)\right\| J, T, M_{T}\right\rangle,
\end{aligned}
$$

\footnotetext{
${ }^{1}$ This definition of $c_{j}^{p}$ and $c_{j}^{n}$ differs by a factor of 2 with respect to the one used in, e.g. [25]. This is consistent with Eq. (2) and our normalization of the nuclear response functions. Our response functions are a factor of 4 larger than those given in output by the Mathematica notebook in [24].
} 
where $\hat{A}_{L ; \tau^{\prime}}(q)$ and $\hat{B}_{L ; \tau^{\prime}}(q)$ can each be one of the nuclear response operators defined below in Eq. (5). There are six independent nuclear response functions where $A=B$ and two interference ones with $B \neq A$. For $B=A$, we simplify the notation writing $W_{A A}^{\tau \tau^{\prime}}=W_{A}^{\tau \tau^{\prime}}$. In Eq. (3), $T$ and $M_{T}$ are the nuclear isospin and associated magnetic quantum number, respectively. Matrix elements in Eq. (3) are reduced in the spin magnetic quantum number $M_{J}$ according to

$$
\begin{aligned}
\left\langle J, M_{J}\left|M_{L M ; \tau}\right| J, M_{J}\right\rangle= & (-1)^{J-M_{J}}\left(\begin{array}{ccc}
J & L & J \\
-M_{J} & M & M_{J}
\end{array}\right) \\
& \times\left\langle J\left\|M_{L ; \tau}\right\| J\right\rangle .
\end{aligned}
$$

The nuclear response operators in Eq. (3) admit the following representation:

$$
\begin{aligned}
M_{L M ; \tau}(q)= & \sum_{i=1}^{A} M_{L M}\left(q \boldsymbol{\rho}_{i}\right) t_{(i)}^{\tau}, \\
\Sigma_{L M ; \tau}^{\prime}(q)= & -i \sum_{i=1}^{A}\left[\frac{1}{q} \vec{\nabla}_{\boldsymbol{\rho}_{i}} \times \mathbf{M}_{L L}^{M}\left(q \boldsymbol{\rho}_{i}\right)\right] \cdot \vec{\sigma}_{(i)} t_{(i)}^{\tau}, \\
\Sigma_{L M ; \tau}^{\prime \prime}(q)= & \sum_{i=1}^{A}\left[\frac{1}{q} \vec{\nabla}_{\boldsymbol{\rho}_{i}} M_{L M}\left(q \boldsymbol{\rho}_{i}\right)\right] \cdot \vec{\sigma}_{(i)} \tau_{(i)}^{\tau}, \\
\Delta_{L M ; \tau}(q)= & \sum_{i=1}^{A} \mathbf{M}_{L L}^{M}\left(q \boldsymbol{\rho}_{i}\right) \cdot \frac{1}{q} \vec{\nabla}_{\boldsymbol{\rho}_{i}} t_{(i)}^{\tau}, \\
\tilde{\Phi}_{L M ; \tau}^{\prime}(q)= & \sum_{i=1}^{A}\left[\left(\frac{1}{q} \vec{\nabla}_{\boldsymbol{\rho}_{i}} \times \mathbf{M}_{L L}^{M}\left(q \boldsymbol{\rho}_{i}\right)\right) \cdot\left(\vec{\sigma}_{(i)} \times \frac{1}{q} \vec{\nabla}_{\boldsymbol{\rho}_{i}}\right)\right. \\
& \left.+\frac{1}{2} \mathbf{M}_{L L}^{M}\left(q \boldsymbol{\rho}_{i}\right) \cdot \vec{\sigma}_{(i)}\right] t_{(i)}^{\tau}, \\
\Phi_{L M ; \tau}^{\prime \prime}(q)= & i \sum_{i=1}^{A}\left(\frac{1}{q} \vec{\nabla}_{\boldsymbol{\rho}_{i}} M_{L M}\left(q \boldsymbol{\rho}_{i}\right)\right) \cdot\left(\vec{\sigma}_{(i)} \times \frac{1}{q} \vec{\nabla}_{\boldsymbol{\rho}_{i}}\right) t_{(i)}^{\tau},
\end{aligned}
$$

where $\boldsymbol{\rho}_{i}$ is the $i$ th nucleon position vector in the nucleus center-of-mass (c.m.) frame and $\vec{\sigma}_{(i)}$ denotes the Pauli spin matrices. In Eq. (5) we define $M_{L M}\left(q \rho_{i}\right)=$ $j_{L}\left(q \boldsymbol{\rho}_{i}\right) Y_{L M}\left(\Omega_{\boldsymbol{\rho}_{i}}\right) \quad$ and $\quad \mathbf{M}_{L L}^{M}\left(q \boldsymbol{\rho}_{i}\right)=j_{L}\left(q \boldsymbol{\rho}_{i}\right) \mathbf{Y}_{L L 1}^{M}\left(\Omega_{\boldsymbol{\rho}_{i}}\right)$, where $\Omega_{\boldsymbol{\rho}_{i}}$ represents azimuthal and polar angles of $\boldsymbol{\rho}_{i}$; $Y_{L M}$ and $\mathbf{Y}_{L L 1}^{M}$ are spherical and vector-spherical harmonics, respectively; and $j_{L}$ are spherical Bessel functions. The nuclear response functions $W_{\ell}^{\tau \tau^{\prime}}$ and $W_{m}^{\tau \tau^{\prime}}$ in Eq. (3) depend on $q$ quadratically when single-nucleon states are expressed in the harmonic oscillator (HO) basis.

The nuclear response operators in Eq. (3) arise from the multipole expansion of nuclear charges and currents produced in the scattering of dark matter by nuclei [9,24]: $M_{L M ; \tau}$ arises from the nuclear vector charge;
$\Sigma_{L M ; \tau}^{\prime}$ and $\Sigma_{L M ; \tau}^{\prime \prime}$ from the nuclear spin current; $\Delta_{L M ; \tau}$ from the nuclear convection current; and $\tilde{\Phi}_{L M ; \tau}^{\prime}$ and $\Phi_{L M ; \tau}^{\prime \prime}$ from the nuclear spin-velocity current. In the zero-momentum transfer limit, a simple intuitive characterization for some of the nuclear response operators in Eq. (3) is possible. For a given target nucleus, $M_{00 ; \tau}$ measures the nucleon content, $\Sigma_{1 M ; \tau}^{\prime}$ and $\Sigma_{1 M ; \tau}^{\prime \prime}$ the nucleon spin content, $\Delta_{1 M ; \tau}$ the distribution of nucleon orbital angular momentum, and $\Phi_{00 ; \tau}^{\prime \prime}$ the nucleon spin-orbit coupling content.

\section{B. $A b$ initio nuclear response functions}

In this work we employ the $a b$ initio no-core shell model (NCSM) technique $[31,66]$ to evaluate the various nuclear response functions in Eq. (3). The starting point of NCSM calculations is the nonrelativistic Hamiltonian for a system of $A$ nucleons interacting by realistic nucleon-nucleon $\left(V_{N N}\right)$ and three-nucleon $\left(V_{N N N}\right)$ interactions:

$$
H=\sum_{i=1}^{A} \frac{\hat{\mathbf{p}}_{i}^{2}}{2 m_{N}}+\sum_{i<j=1}^{A} \hat{V}_{N N ; i j}+\sum_{i<j<k=1}^{A} \hat{V}_{N N N ; i j k},
$$

where $\mathbf{p}_{i}$ are the nucleon momenta. In NCSM, the total wave function is expanded and the Hamiltonian is diagonalized in a fully antisymmetric many-body $\mathrm{HO}$ basis.

In the present study we focus on few-body nuclear systems. In this case it is most efficient to formulate NCSM in relative Jacobi coordinates [66]. Different sets of Jacobi coordinates can be employed, one of which is particularly suitable for the construction of the antisymmetrized $\mathrm{HO}$ basis:

$$
\begin{aligned}
\boldsymbol{\xi}_{0} & =\sqrt{\frac{1}{A} \sum_{j=1}^{A} \mathbf{r}_{j},} \\
\boldsymbol{\xi}_{i} & =\sqrt{\frac{i}{i+1}}\left(\frac{1}{i} \sum_{j=1}^{i} \mathbf{r}_{j}-\mathbf{r}_{i+1}\right),
\end{aligned}
$$

with $\mathbf{r}_{i}$ being the coordinate of nucleon $i=1, \ldots, A$. In this set, graphically represented in Fig. 1 for $A=4$ nucleons, the coordinate $\xi_{0}$ is proportional to the c.m. coordinate of the $A$-body system and the coordinates $\xi_{i}, i=1, \ldots, A-1$, are proportional to the relative positions of nucleon $i+1$ with respect to the c.m. of the $i$-nucleon subcluster. When the single-nucleon coordinates and momenta in the Hamiltonian (6) are transformed into coordinates (7), the kinetic term splits into a part depending only on the c.m. coordinate $\xi_{0}$ and an intrinsic part depending only on the internal Jacobi coordinates $\left\{\boldsymbol{\xi}_{i}\right\}_{i=1}^{A-1}$. Translational invariance of $V_{N N}$ and $V_{N N N}$ interactions, i.e. independence of $\boldsymbol{\xi}_{0}$, allows us to separate out the c.m. term and thus decrease the number of degrees of freedom. Consequently, the $A$-body $\mathrm{HO}$ basis states can be constructed as 


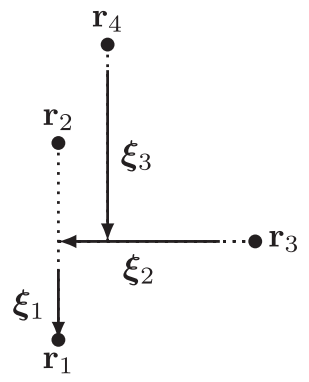

FIG. 1. Graphical representation of the relative Jacobi coordinates defined in Eq. (7) for $A=4$ nucleons.

$$
\left.\left.\mid\left(\ldots\left(\alpha_{1}, \alpha_{2}\right) J_{3} T_{3}, \alpha_{3}\right) J_{4} T_{4}, \ldots, \alpha_{A-1}\right) J_{A} T_{A}\right\rangle,
$$

where $\left|\alpha_{i}\right\rangle \equiv\left|n_{i}\left(l_{i} s_{i}\right) j_{i} t_{i}\right\rangle$ are HO states, depending on coordinates $\xi_{i}$, with radial $n_{i}$, orbital $l_{i}$, spin $s_{i}$, angular momentum $j_{i}$, and isospin $t_{i}$ quantum numbers. The parentheses in (8) represent angular momentum and isospin coupling. The quantum numbers $J_{i}$ and $T_{i}(i=3, \ldots, A)$ are angular momentum and isospin quantum numbers of $i$ nucleon clusters. The basis is truncated by restricting the total number of $\mathrm{HO}$ quanta:

$$
\sum_{i} 2 n_{i}+l_{i} \leq N_{\max }
$$

which defines the size of the model space. NCSM calculations are thus variational and converge to exact results with increasing $N_{\max }$. In the case of few-body systems, as considered in this work, calculations with sufficiently large $N_{\text {max }}$ can be performed to reach satisfactory convergence.

Before the diagonalization of the Hamiltonian (6) the basis states (8) have to be antisymmetrized with respect to the exchanges of all nucleons. The antisymmetrization procedure with Jacobi-coordinate $\mathrm{HO}$ basis states is extensively discussed in Ref. [66] and we will only summarize its main points here. The fully antisymmetric A-body $\mathrm{HO}$ basis is obtained by diagonalization of the antisymmetrizer operator $\hat{\mathcal{A}}_{A}$ between the basis states (8). The antisymmetrizer is defined as

$$
\hat{\mathcal{A}}_{A}=\frac{1}{A !} \sum_{\pi} \operatorname{sgn}(\pi) \hat{\mathcal{P}}_{\pi}
$$

where the summation extends over all permutations $\pi$, with parity $\operatorname{sgn}(\pi)$, of single-nucleon coordinates realized on the states (8) by permutation operator $\hat{\mathcal{P}}_{\pi}$. The eigenvectors of the antisymmetrizer (10) span two eigenspacesone corresponding to eigenvalue 1 formed by physical antisymmetric states and one corresponding to eigenvalue 0 formed by spurious states. The antisymmetrizer can be represented as

$$
\hat{\mathcal{A}}_{A}=\hat{\mathcal{A}}_{A-1} \frac{1}{A}\left[1-(A-1) \hat{\mathcal{P}}_{A-1, A}\right] \hat{\mathcal{A}}_{A-1},
$$

where the operator $\hat{\mathcal{P}}_{A-1, A}$ interchanges the coordinates of nucleons $A-1$ and $A$. Equation (11) provides the basis for an iterative procedure to obtain fully antisymmetrized states from states with a lower degree of antisymmetry. Explicit expressions for the matrix elements of the antisymmetrizer $\hat{\mathcal{A}}_{A}$ between the basis states (8) can be found e.g. in Ref. [67]. The resulting states can be expanded in terms of the original basis containing an antisymmetric cluster of $A-1$ nucleons and one nucleon as

$$
\begin{aligned}
& \left|N_{A} i_{A} J_{A} T_{A}\right\rangle \\
& \quad=\sum\left\langle\left(N_{A-1} i_{A-1} J_{A-1} T_{A-1}, \alpha_{A-1}\right) J_{A} T_{A} \mid N_{A} i_{A} J_{A} T_{A}\right\rangle \\
& \quad \times\left|\left(N_{A-1} i_{A-1} J_{A-1} T_{A-1}, \alpha_{A-1}\right) J_{A} T_{A}\right\rangle,
\end{aligned}
$$

where the expansion coefficients obtained from the eigenvectors of the antisymmetrizer are the coefficients of fractional parentage. Thanks to the important property of the antisymmetrizer of being diagonal in the total number of HO quanta $N_{A}$ the fully antisymmetric states (12) can be classified by $N_{A}=N_{A-1}+2 n_{A-1}+l_{A-1}\left(N_{2}=2 n_{1}+l_{1}\right.$ for two-nucleon states) and the quantum number $i_{A}$ which distinguishes different states with the same set of quantum numbers $N_{A}, J_{A}, T_{A}$.

To evaluate the matrix elements of two- and three-body $V_{N N}$ and $V_{N N N}$ potentials in the Hamiltonian (6) between the antisymmetrized many-body $\mathrm{HO}$ states one can recursively make use of the expansion in Eq. (12). However, it is more efficient to employ more suitable sets of Jacobi coordinates together with the associated $\mathrm{HO}$ states containing antisymmetrized states of $A-2$ and two nucleons or $A-3$ and three nucleons [66].

To construct the nuclear response functions defined in Eq. (3) we need to evaluate matrix elements of the various operators in Eq. (5) between the ground-state wave functions of the Hamiltonian. The nuclear matrix elements in (3) can be further reduced in nuclear isospin and written as

$$
\begin{array}{r}
\left\langle J^{\pi} T M_{T}\left\|\sum_{i=1}^{A} \hat{A}_{L \tau}\left(q \boldsymbol{\rho}_{i}\right)\right\| J^{\pi} T M_{T}\right\rangle \\
=(-1)^{T-M_{T}}\left(\begin{array}{ccc}
T & \tau & T \\
-M_{T} & 0 & M_{T}
\end{array}\right) \\
\quad \times\left\langle J^{\pi} T|\|| \sum_{i=1}^{A} \hat{A}_{L \tau}\left(q \boldsymbol{\rho}_{i}\right)|| \mid J^{\pi} T\right\rangle .
\end{array}
$$

The NCSM technology for computing such nuclear matrix elements is analogous to standard SM calculations. One-body transition densities (OBTD) are introduced so that the many-body matrix elements of one-body operators (reduced both in nuclear spin and isospin) can be expressed as products of OBTD and single-particle matrix elements [67]: 


$$
\begin{aligned}
& \left\langle J^{\pi} T\left|\| \sum_{i=1}^{A} \hat{A}_{L \tau}\left(q \rho_{i}\right)\right||| J^{\pi} T\right\rangle \\
& =\sum_{\alpha \beta} \Psi_{\alpha \beta}^{L \tau}\left\langle\alpha \left|\left\|\hat{A}_{L \tau}\left(q \rho_{A}\right)|\| \beta\rangle,\right.\right.\right.
\end{aligned}
$$

where $|\alpha(\beta)\rangle=\left|n_{\alpha(\beta)}\left(l_{\alpha(\beta)} \frac{1}{2}\right) j_{\alpha(\beta)} \frac{1}{2}\right\rangle$ denote single-nucleon HO states associated with Jacobi coordinate $\xi_{A-1}=$ $-\sqrt{A /(A-1)} \rho_{A}$. The OBTD $\Psi_{\alpha \beta}^{L \tau}$ is given by

$$
\begin{aligned}
\Psi_{\alpha \beta}^{L \tau}= & A \sum\left\langle J^{\pi} T \mid\left(N_{A-1} i_{A-1} J_{A-1} T_{A-1}, \alpha\right) J T\right\rangle \\
& \times\left\langle\left(N_{A-1} i_{A-1} J_{A-1} T_{A-1}, \beta\right) J T \mid J^{\pi} T\right\rangle \\
& \times \hat{J}^{2}(-1)^{J_{A-1}+L+J+j_{\beta}}\left\{\begin{array}{ccc}
J_{A-1} & j_{\beta} & J \\
L & J & j_{\alpha}
\end{array}\right\} \\
& \times \hat{T}^{2}(-1)^{T_{A-1}+\tau+T+\frac{1}{2}}\left\{\begin{array}{ccc}
T_{A-1} & \frac{1}{2} & T \\
\tau & T & \frac{1}{2}
\end{array}\right\},
\end{aligned}
$$

where the terms in curly brackets are the Wigner $6 j$ symbols and we used expansion of the eigenstate in the basis (12). In Eq. (14), the single-particle matrix elements reduced in both angular momentum and isospin can be simplified by using $\left\langle\frac{1}{2}\left\|t^{\tau}\right\| \frac{1}{2}\right\rangle=\sqrt{2(2 \tau+1)}$ :

$$
\left\langle\alpha||\left|\hat{O}_{L \tau}\left(q \rho_{A}\right)\right||| \beta\right\rangle=\sqrt{2(2 \tau+1)}\left\langle\alpha\left\|\hat{O}_{L}\left(q \rho_{A}\right)\right\| \beta\right\rangle,
$$

where $\left\langle\alpha\left\|\hat{O}_{L}\left(q \boldsymbol{\rho}_{A}\right)\right\| \mid \beta\right\rangle$ are single-particle matrix elements reduced in angular momentum only. In a $\mathrm{HO}$ basis these matrix elements can be calculated analytically and are listed e.g. in Ref. [9].

\section{Chiral nuclear interactions}

The theory of nuclear forces has a long history—starting with the seminal meson-exchange hypothesis of Yukawa. The current state of the art involves the use of chiral EFT and has opened the door for a description of atomic nuclei consistent with the underlying symmetries of QCD. Nuclear interactions from chiral EFT are based on the use of nucleons and pions as the relevant degrees of freedom, but employ symmetries and the pattern of spontaneous symmetry breaking of QCD [16-18]. In this approach, the exchange of pions within chiral perturbation theory yields the long-ranged contributions of the nuclear interaction, while short-ranged components are included as contact terms. Regularization is needed to deal with divergent momentum-space integrals. The interaction is parametrized in terms of low-energy constants (LECs) that, in principle, can be connected to QCD predictions. However, the currently viable approach to accurately describe atomic nuclei in chiral EFT requires that the LECs are constrained from experimental low-energy data. The bulk of this fit data traditionally consists of cross sections measured in nucleon-nucleon scattering experiments. The interactions from chiral EFT exhibit a power counting in the ratio $Q / \Lambda$, with $Q$ being the lowmomentum scale that is characteristic for the nuclear observable under consideration and $\Lambda$ the EFT breakdown scale, which is of the order of $1 \mathrm{GeV}$. In this approach, three-nucleon forces enter at next-to-next-to-leading order (NNLO). Both regulator independence and an accurate power counting scheme are crucial ingredients for the EFT approach to nuclear forces. In combination these properties allow for order-by-order improvement with decreasing truncation error, where the magnitude of such errors can also be quantified.

In this work, the nuclear interaction enters in the manybody Hamiltonian (6) that is diagonalized in a basis to yield the nuclear wave function. In order to capitalize on recent developments in the quantification of uncertainties of nuclear forces $[39,68-70]$ we employ the family of 42 different interactions at NNLO (labeled $\mathrm{NNLO}_{\text {sim }}$ ) that was constructed by Carlsson et al. [39]. These potentials are optimized to simultaneously reproduce $N N$ as well as $\pi N$ scattering data, the binding energies and charge radii of

${ }^{2,3} \mathrm{H}$ and ${ }^{3} \mathrm{He}$, the quadrupole moment of ${ }^{2} \mathrm{H}$, as well as the $\beta$-decay half-life of ${ }^{3} \mathrm{H}$. Utilizing such a large set of interactions allows us to better explore the systematic uncertainties. Each $\mathrm{NNLO}_{\text {sim }}$ potential is associated with one of seven different regulator cutoffs $\Lambda_{\mathrm{EFT}}=$ $450,475, \ldots, 575,600 \mathrm{MeV}$. In addition, the database of experimental $N N$ scattering cross sections used to constrain the respective interaction was also varied. It was truncated at six different maximum scattering energies in the laboratory system $T_{\mathrm{Lab}}^{\max }=125, \ldots, 290 \mathrm{MeV}$. It should be pointed out that for all $\mathrm{NNLO}_{\text {sim }}$ interactions an equally good description of the fit data is attained and that all LECs are of natural size. See Ref. [39] for a complete description. In this work we are mainly interested in the nuclear wave functions of ${ }^{3,4} \mathrm{He}$. We note that the binding energy of ${ }^{3} \mathrm{He}$ is accurately described for all these interactions since it is included in the pool of fit data. Predictions for $E\left({ }^{4} \mathrm{He}\right)$ vary within a $\sim 2 \mathrm{MeV}$ range around the experimental binding energy.

\section{RESULTS}

The main focus of this work is to quantify the impact of systematic nuclear structure uncertainties on the interpretation of data from dark matter searches. In the present study we consider only light nuclear systems that can be calculated reliably and accurately without uncontrollable approximations. In particular, we performed $a b$ initio NCSM calculations of ${ }^{3} \mathrm{He}$ and ${ }^{4} \mathrm{He}$ and constructed all relevant nuclear response functions that appear in elastic dark matter-nucleus scattering. The generated response functions were then employed to explore the sensitivity of selected physical observables to nuclear-structure uncertainties. 


\section{A. Nuclear response functions of ${ }^{3} \mathrm{He}$ and ${ }^{4} \mathrm{He}$}

In this section we present the nuclear response functions defined in Eq. (3). In order to evaluate the nuclear matrix elements in Eq. (14) and construct the nuclear response functions we performed $a b$ initio NCSM calculations of ${ }^{3} \mathrm{He}$ and ${ }^{4} \mathrm{He}$ ground-state wave functions using the complete family of all $42 \mathrm{NNLO}_{\text {sim }}$ chiral nuclear Hamiltonians. The NCSM model spaces used in these calculations are very large, $N_{\max }=40(20)$ for ${ }^{3} \mathrm{He}\left({ }^{4} \mathrm{He}\right)$, so that both energies and wave functions are fully converged. The systematic uncertainties in the determination of the underlying $N N$ and $N N N$ interactions are probed through the use of a large family of interactions. These uncertainties propagate into the set of calculated nuclear wave functions and thus manifest themselves as uncertainties in the determination of the nuclear response functions. The types of the nuclear responses generated by a particular nucleus depend on the total nuclear ground-state angular momentum and isospin as well as on the details of the nuclear structure.

In the case of ${ }^{4} \mathrm{He}$ most of the response functions are identically zero due to the $J=0$ and $T=0$ ground-state quantum numbers. The only nonvanishing nuclear response functions are the isoscalar spin-independent $W_{M}^{00}, W_{\Phi^{\prime \prime}}^{00}$ and $W_{\Phi^{\prime \prime} M}^{00}$ responses, which are shown in Fig. 2 as functions of the transferred (recoil) momentum $q$. All $\mathrm{NNLO}_{\text {sim }}$ chiral nuclear Hamiltonians were used to calculate a ${ }^{4} \mathrm{He}$ groundstate wave function and to evaluate the response functions. These different curves turn out to be evenly distributed in regions that are then represented by shaded bands in the figures. In that way, the response functions calculated with the $a b$ initio NCSM technique reflect the systematic uncertainty in the underlying nuclear interaction. The nuclear-structure uncertainties affect the various response functions very differently. While the dominant nuclear response $W_{M}^{00}$ is determined fairly accurately, the $W_{\Phi^{\prime \prime}}^{00}$ and $W_{\Phi^{\prime \prime} M}^{00}$ responses suffer from large uncertainties, in particular in the region of low recoil momenta $q \approx 0 \mathrm{GeV}$. It is to be noted that $W_{\Phi^{\prime \prime}}^{00}$ and $W_{\Phi^{\prime \prime} M}^{00}$ appear suppressed by a factor of $q^{2} / m_{N}^{2}$ in the scattering cross section (2) and the large uncertainties are thus suppressed in the physical observables, as will be demonstrated in Sec. III B. The large uncertainties found in the $W_{\Phi^{\prime \prime}}^{00}$ (and consequently $W_{\Phi^{\prime \prime} M}^{00}$ ) response compared to $W_{M}^{00}$ can be understood by examining the long-wavelength limit $(q \rightarrow 0)$ of the leading multipoles of the corresponding nuclear operators [9]. In this limit we have

$$
M_{00 ; 0}(q) \stackrel{q \rightarrow 0}{\rightarrow} \frac{1}{\sqrt{4 \pi}} \sum_{i=1}^{A} \mathbb{1}_{(i)},
$$

which implies that the $W_{M}^{00}(q \rightarrow 0)$ response is proportional to $A^{2}$ independent of the nuclear dynamics. On the other hand, since

$$
\Phi_{00 ; 0}^{\prime \prime}(q) \stackrel{q \rightarrow 0}{\rightarrow} \frac{-1}{3 \sqrt{4 \pi}} \sum_{i=1}^{A} \boldsymbol{\sigma}_{(i)} \cdot \mathbf{l}_{(i)}
$$

the $W_{\Phi^{\prime \prime}}^{00}(q \rightarrow 0)$ response is proportional to the square of the expectation value of the nucleon spin-orbit coupling in the nuclear ground state. This quantity is difficult to access experimentally and its value is therefore not constrained. Consequently, the $q \rightarrow 0$ behavior of the $W_{\Phi^{\prime \prime}}^{00}$ and $W_{\Phi^{\prime \prime} M}^{00}$ responses are predictions of the nuclear model, clearly very sensitive to the underlying nuclear Hamiltonian.

Furthermore, the functional dependence of the nuclear response functions on the recoil momentum $q$ can be understood from expressions (3) and (14). In a HO basis the single-particle matrix elements in Eq. (14) can be evaluated analytically, yielding an expression of the form $P(y) \mathrm{e}^{-y}$, where $P(y)$ is a polynomial [9] and $y=(q b / 2)^{2}$ a dimensionless quantity with $b=\sqrt{\hbar /\left(m_{N} \omega\right)}$ the $\mathrm{HO}$ length. Since the one-body transition densities are independent of $q$, the nuclear response functions follow this exponential suppression and their absolute uncertainties decrease with $q$. On the other hand, the relative uncertainties in the response functions increase for larger values of recoil momentum. For comparison, also shown in Fig. 2 is the $W_{M}^{00}$ response function taken from Ref. [25] in which SM calculations were performed for a number of light elements in the context of dark matter-nucleus scattering. In the particular cases of ${ }^{3,4} \mathrm{He}$, the interaction that was used in that work did not allow coupling between the $0 s_{1 / 2}$ shell and higher-lying orbits. As a consequence, the ground-state configuration is a single Slater determinant with all four nucleons in the $0 s_{1 / 2}$ shell implying that the results

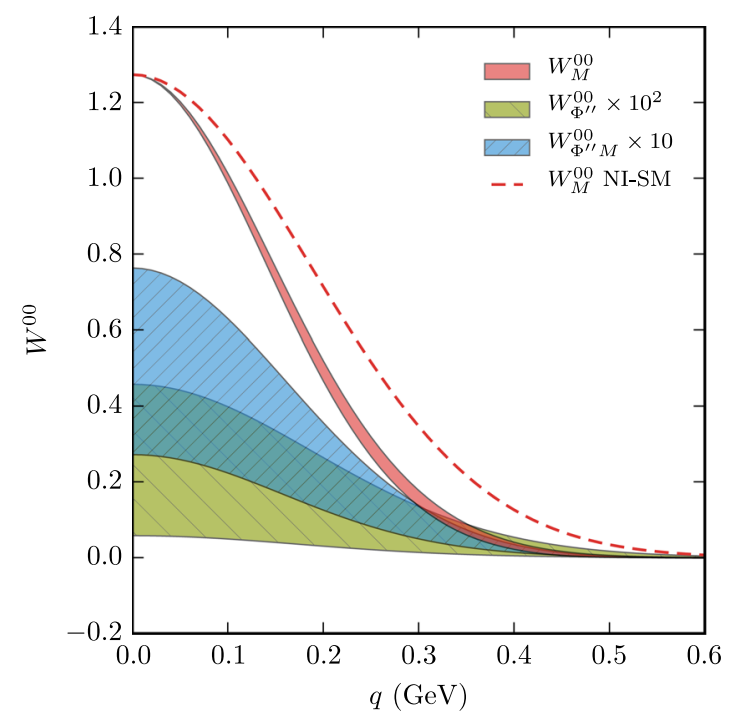

FIG. 2. Isoscalar nuclear response functions $W_{M}^{00}, W_{\Phi^{\prime \prime}}^{00}$ and $W_{\Phi^{\prime \prime} M}^{00}$ of ${ }^{4} \mathrm{He}$ as functions of the transferred momentum $q$, calculated within $a b$ initio NCSM (shaded regions) and the noninteracting shell model (NI-SM) (dashed line). 
correspond to a noninteracting shell model (NI-SM). Even though this situation is an extreme limiting case for the SM it is worth pointing out the main differences and new features of $a b$ initio calculations. In general, the $W_{M}^{00}$ response functions generated within the SM approach using phenomenological interactions and the NCSM approach using chiral EFT interactions will match for recoil momentum $q=0 \mathrm{GeV}$, due to the $A^{2}$-normalization, and for large values of $q$, where the response must vanish. Except for these limits, the results will differ. In particular, for ${ }^{4} \mathrm{He}$ the NI-SM give larger values of $W_{M}^{00}$ compared to NCSM calculations. Moreover, it is to be stressed that $W_{M}^{00}$ is the only nonzero nuclear response function resulting from the NI-SM calculation, as it includes only one single-particle orbital. In general, SM calculations with residual valence space interactions will employ a very restricted single-particle basis. The calculation of the nuclear response should therefore be made with operators that have been properly renormalized to act only within this truncated model space. In contrast, the nuclear responses in Fig. 2 calculated within the NCSM methodology were obtained employing a substantially larger model space. The NCSM method allows us to systematically increase the size of the model space. For these calculations a truncation of $N_{\max }=20$ was used to reach full convergence with the use of bare operators. The NCSM model space is able to accommodate details of the nuclear structure that are crucial to expose the full complexity of the nuclear response. This difference becomes even more evident for the $W_{\Phi^{\prime \prime}}^{00}$ response, which is evaluated as zero in the restricted $N_{\max }=0\left(0 s_{1 / 2}\right)$ model space but is nonzero in the NCSM as it receives contributions from nucleons that occupy higher orbitals.

Similar conclusions hold for the nuclear response functions of ${ }^{3} \mathrm{He}$ as shown in Figs. 3 and 4. In this case there are more nonvanishing response functions due to the $J=\frac{1}{2}$ and $T=\frac{1}{2}$ ground-state quantum numbers. In particular, among all the response functions in Eq. (3) only the $W_{\tilde{\Phi}^{\prime}}^{\tau \tau^{\prime}}$ response vanishes, since it contributes for nuclei with total angular momentum $J \geq 1$. The dominant nuclear responses of ${ }^{3} \mathrm{H}$, resulting both from ab initio NCSM calculations and the NI-SM [25], are the spin-independent responses $W_{M}^{\tau \tau^{\prime}}$, shown in Fig. 3, and the spin-dependent response functions $W_{\Sigma^{\prime}}^{\tau \tau^{\prime}}$ and $W_{\Sigma^{\prime \prime}}^{\tau \tau^{\prime}}$, shown in the left panel of Fig. 4. The nuclear structure uncertainties in the determination of the $W_{M}^{\tau \tau^{\prime}}$ response are negligibly small, making the corresponding bands in Fig. 3 almost invisible. As in the case of ${ }^{4} \mathrm{He}$, the ab initio NCSM $W_{M}^{\tau \tau^{\prime}}$ response functions are smaller than the ones from the NI-SM over the whole range of relevant recoil momenta, except for $q \rightarrow 0$ where they must agree due to the $A^{2}$-normalization. The spin-dependent responses $W_{\Sigma^{\prime}}^{\tau \tau^{\prime}}$ and $W_{\Sigma^{\prime \prime}}^{\tau \tau^{\prime}}$ are generated by the $\Sigma_{L M ; \tau}^{\prime}$ and $\Sigma_{L M ; \tau}^{\prime \prime}$ nuclear operators whose leading multipoles are proportional to the

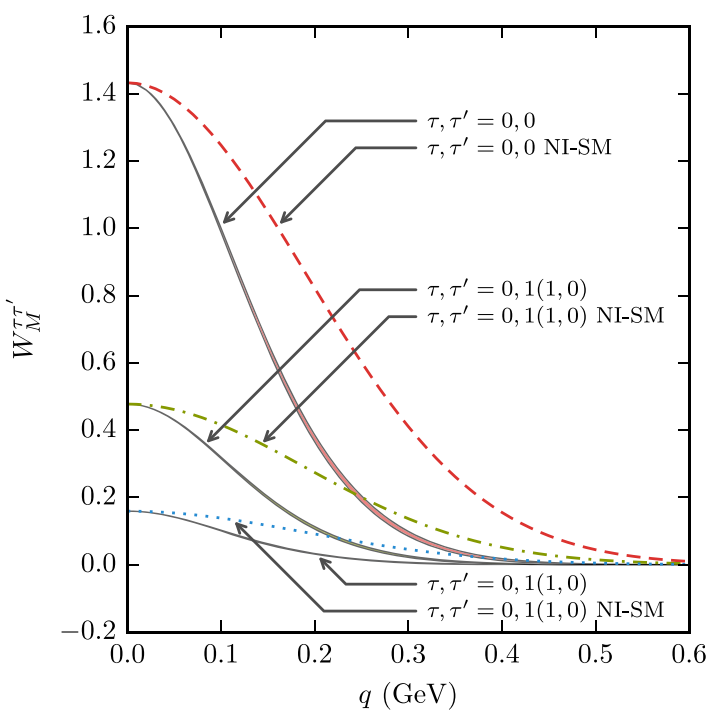

FIG. 3. Nuclear response functions $W_{M}^{\tau \tau^{\prime}}$ of ${ }^{3} \mathrm{He}$ as functions of the transferred momentum $q$, calculated within the $a b$ initio NCSM (shaded regions) and the NI-SM (dashed, dashed-dotted and dotted lines).

total nuclear spin operator in the $q \rightarrow 0$ limit [9]. Similarly as for the $\Phi_{L M ; \tau}^{\prime \prime}$ operator, the ground-state expectation value of the nuclear spin, $\frac{1}{2} \sum_{i=1}^{A} \vec{\sigma}_{(i)}$, is not imposed as a strict constraint and its value can vary for different nuclear Hamiltonians. However, the nuclear uncertainties affect these response functions only moderately. The $a b$ initio NCSM calculations generate additional nuclear responses not appearing in the NI-SM, namely the $W_{\Phi^{\prime \prime}}^{\tau \tau^{\prime}}$ and $W_{\Delta}^{\tau \tau^{\prime}}$ response functions. These, in turn, generate the interference responses $W_{\Phi^{\prime \prime} M}^{\tau \tau^{\prime}}$ and $W_{\Delta \Sigma^{\prime}}^{\tau \tau^{\prime}}$. Finally, the leading multipole of the nuclear response operator $\Delta_{L M ; \tau}$ is proportional to the total nuclear angular momentum, $\sum_{i=1}^{A} \vec{l}_{(i)}$ [9], and its expectation value is also not imposed as a constraint on the nuclear Hamiltonian. Consequently, as shown in Fig. 4, all these response functions exhibit large systematic uncertainties that are, however, suppressed in physical observables by a factor of $q^{2} / m_{N}^{2}$. Furthermore, isovector responses are generally smaller in magnitude since they result from a proton-neutron difference rather than a sum of proton and neutron contributions.

\section{B. Impact on dark matter searches}

Nuclear physics uncertainties in the response functions affect the interpretation of data from dark matter search experiments. In this subsection we quantitatively address this matter in the context of directional dark matter detection for which detectors with ${ }^{3,4} \mathrm{He}$ target materials are currently in a research and development stage. The aim is to assess the impact of nuclear physics uncertainties for these isotopes on physical observables.

We start by reviewing the basic concepts of directional dark matter detection. The Earth's motion in the galactic 

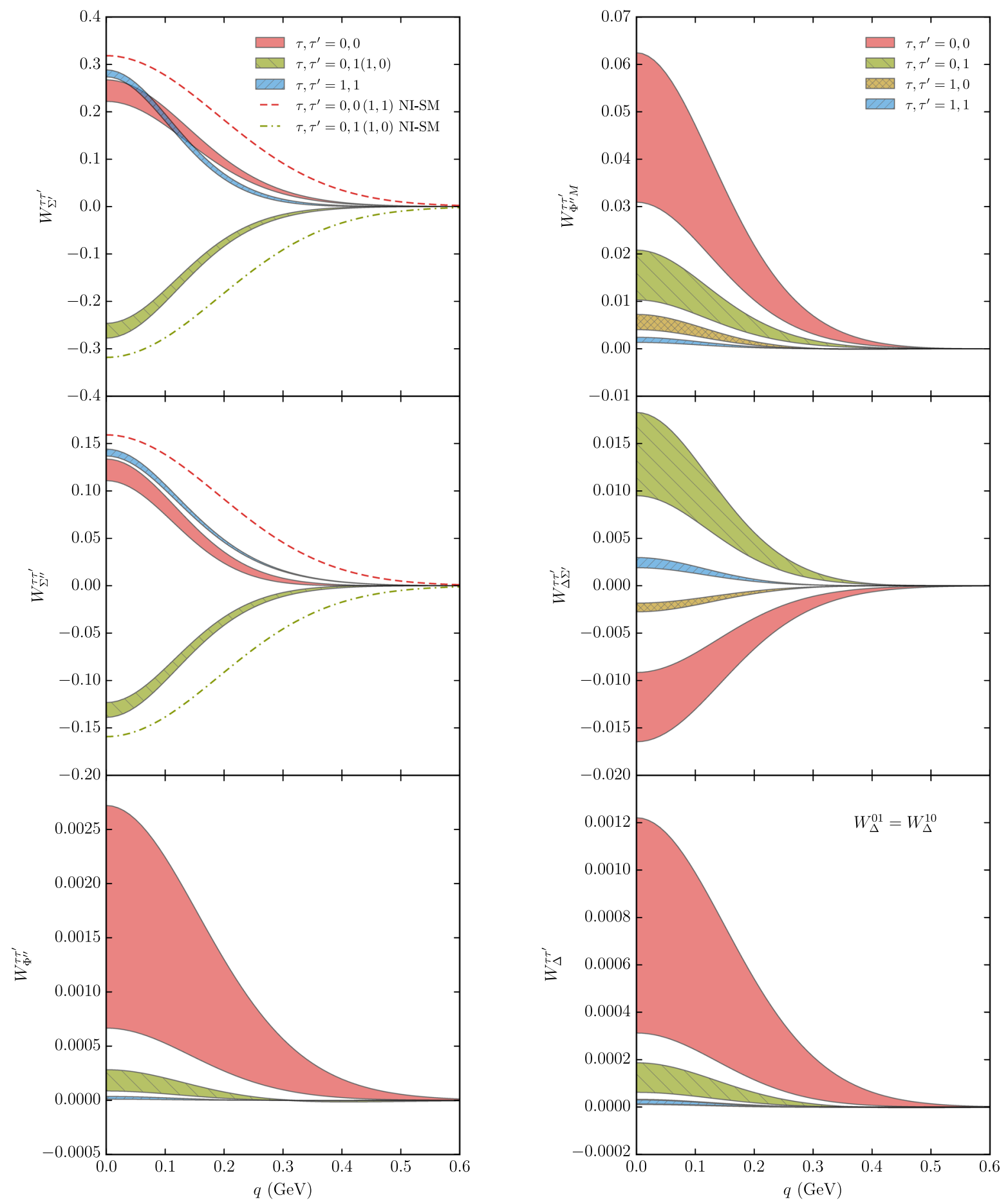

FIG. 4. Nuclear response functions of ${ }^{3} \mathrm{He}$ as functions of the transferred momentum $q$ calculated within $a b$ initio NCSM (shaded regions) and NI-SM (dashed and dashed-dotted lines).

rest frame induces a flux of dark matter particles across the surface of the planet. If dark matter interacts with nuclei, low-background experiments might be able to detect nuclear recoils induced by the scattering of dark matter particles in a target material [71]. The angular distribution of such nuclear recoil events is expected to be anisotropic, as the Earth's motion in the galactic rest frame selects a preferred direction in the sphere of recoil directions [72]. Depending on the interaction operator in analysis, recoil events are mainly expected in the direction opposite to the observer's motion, or in a ring around it $[60,61]$. In order to exploit this information, directional dark matter detectors have been designed. They search for anisotropies in the distribution of nuclear recoil events in low-background 
underground experiments. Here we consider hypothetical detectors made of ${ }^{3} \mathrm{He}$ or ${ }^{4} \mathrm{He}$.

Let us now focus on physical observables. The double differential rate of nuclear recoil events per unit detector mass is given by

$$
\begin{aligned}
\frac{\mathrm{d}^{2} \mathcal{R}}{\mathrm{d} q^{2} \mathrm{~d} \Omega}= & \alpha \int \mathrm{d}^{3} \mathbf{v} \delta\left(\mathbf{v} \cdot \mathbf{w}-w_{q}\right) f\left(\mathbf{v}+v_{\oplus}(t)\right) \\
& \times v^{2} \frac{\mathrm{d} \sigma\left(v^{2}, q^{2}\right)}{\mathrm{d} q^{2}}
\end{aligned}
$$

where $\alpha=\rho_{\chi} /\left(2 \pi m_{\chi} m_{A}\right), \rho_{\chi}=0.4 \mathrm{GeV} \mathrm{cm}^{-3}$ [73] is the local dark matter density, $m_{A}$ the target nucleus mass, $\mathbf{w}$ a unit vector pointing in the nuclear recoil direction, $w_{q}=q /\left(2 \mu_{\chi A}\right)$ the minimum velocity required to transfer a momentum $q$ in the scattering, and $\mathbf{v}_{\oplus}(t)$ the timedependent Earth's velocity in the galactic rest frame. From now onwards, we assume azimuthal symmetry around the direction of $\mathbf{v}_{\oplus}(t)$, i.e. $\mathrm{d} \Omega=2 \pi \mathrm{d} \cos \theta$, and measure the angle $\theta$ with respect to $\mathbf{v}_{\oplus}(t)$. We approximate the velocity distribution $f\left(\mathbf{v}+\mathbf{v}_{\oplus}(t)\right)$ with a Gaussian function truncated at an escape velocity of $533 \mathrm{~km} \mathrm{~s}^{-1}$, and assume a local standard of rest of $220 \mathrm{~km} \mathrm{~s}^{-1}[74,75]$. The velocity integral in Eq. (19) is a Radon transform. In the Gaussian approximation, it has been evaluated analytically for all operators in Table I in Ref. [60]. The key physical observable for the present analysis is the differential rate of nuclear recoil events per unit detector mass. This can be calculated from Eq. (19) as follows:

$$
\frac{\mathrm{d} \mathcal{R}}{\mathrm{d} \cos \theta}=2 \pi \int_{q^{2}>q_{\mathrm{th}}^{2}} \frac{\mathrm{d}^{2} \mathcal{R}}{\mathrm{d} q^{2} \mathrm{~d} \Omega} \mathrm{d} q^{2}
$$

where $E_{\mathrm{th}} \equiv q_{\mathrm{th}}^{2} /\left(2 m_{A}\right)$ is the detector energy threshold. Here we set $E_{\mathrm{th}}=0$, and assume infinite energy and angular resolution.

We now evaluate Eq. (20) for selected interaction operators, namely, $\hat{\mathcal{O}}_{4}, \hat{\mathcal{O}}_{5}, \hat{\mathcal{O}}_{9}, \hat{\mathcal{O}}_{10}, \hat{\mathcal{O}}_{12}$ and $\hat{\mathcal{O}}_{15}$. The operator $\hat{\mathcal{O}}_{4}$ is the standard spin-dependent interaction. It arises as the leading interaction operator from the nonrelativistic reduction of renormalizable Lagrangians for spin $1 / 2$ or 1 dark matter interacting with nucleons through the exchange of a heavy spin- 1 particle. The operator $\hat{\mathcal{O}}_{5}$ can only be generated as the leading interaction operator if dark matter has spin 1 and interacts with nucleons through the exchange of a heavy spin-1 particle. In contrast, the operator $\hat{\mathcal{O}}_{10}$ can arise for all dark matter particle spins, including spin 0 . Finally, the operator $\hat{\mathcal{O}}_{12}$ is always generated in association with the operator $\hat{\mathcal{O}}_{1}$. We refer to [47] for a comprehensive list of scenarios. Some of the considerations above might be affected by operator evolution [76,77].
In the following we will focus on the contribution of the isoscalar $(\tau=0)$ or isovector $(\tau=1)$ component of a single operator $\hat{\mathcal{O}}_{j} t^{\tau}$ at a time by setting only the corresponding coupling constant $c_{j}^{\tau}$ different from zero. In this case the value of the coupling constant is $c_{j}^{\tau}=10^{-3} / \mathrm{m}_{V}^{2}$, where $m_{V}=246.2 \mathrm{GeV}$ is the electroweak scale. The value $10^{-3} / m_{V}^{2}$ is arbitrary and corresponds to the reference WIMP-nucleon cross section $\left(\mu_{\chi N}^{2} / m_{V}^{4}\right) /(4 \pi) \sim$ $7 \times 10^{-45} \mathrm{~cm}^{2}$ at $m_{\chi}=50 \mathrm{GeV}$, with $\mu_{\chi N}$ the WIMP-nucleon reduced mass. Since the rate depends quadratically on the coupling constants, the results can be easily rescaled to other values of $c_{j}^{\tau}$.

Figure 5 shows the differential rate of nuclear recoil events, Eq. (20), as a function of the recoil direction $\cos \theta$. In the figure we focus on the isoscalar component of selected interaction operators, and consider ${ }^{3} \mathrm{He}$ as a target material. The dark matter particle mass has been set to $m_{\chi}=10 \mathrm{GeV}$ and the coupling constants of the three operators in the legend to the reference value as specified above. For the nuclear response functions needed in this calculation, Eq. (3), we consider two distinct choices, corresponding to lower and upper boundary of the associated nuclear physics uncertainty band found in Sec. III A. This produces the colored bands reported in the figure. They describe the impact of nuclear physics uncertainties

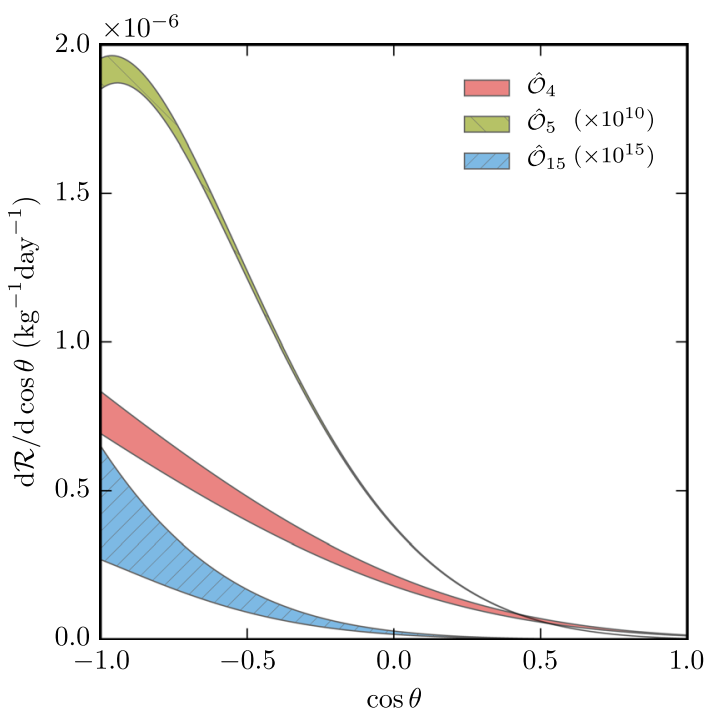

FIG. 5. Differential rate of nuclear recoil events as a function of the recoil direction. We focus on the isoscalar component of the interaction operators listed in the legend and consider ${ }^{3} \mathrm{He}$ as a target material. We set $m_{\chi}=10 \mathrm{GeV}$ and the isoscalar coupling constants to the reference value as detailed in the text. For the nuclear response functions needed in this calculation, Eq. (3), we consider two distinct choices, corresponding to the lower and upper boundaries of the associated nuclear physics uncertainty band found in Sec. II B. This produces the colored bands in the figure, which therefore account for the nuclear physics uncertainties in the recoil rates. 
on the physical observable considered in this investigation. Specifically, in Fig. 5 we consider the following interactions: the operator $\hat{\mathcal{O}}_{4}$, which generates the $W_{\Sigma^{\prime}}^{\tau \tau^{\prime}}$ and $W_{\Sigma^{\prime \prime}}^{\tau \tau^{\prime}}$ responses; the operator $\hat{\mathcal{O}}_{5}$, which generates $W_{M}^{\tau \tau^{\prime}}$ as the leading response; and, finally, the operator $\hat{\mathcal{O}}_{15}$, which as the leading response generates $W_{\Phi^{\prime \prime}}^{\tau \tau^{\prime}}$. In agreement with Sec. III A, nuclear physics uncertainties are large for $\hat{\mathcal{O}}_{15}$, moderate for $\hat{\mathcal{O}}_{4}$, and small for $\hat{\mathcal{O}}_{5}$. For a given interaction operator, the leading response function can be determined from Eq. (A1) and the results in Sec. III A.

Figure 5 also shows that nuclear physics uncertainties are more pronounced at $\cos \theta=-1$, where nuclear recoil rates are large. Around this direction the integral in Eq. (20) is dominated by small values of $q$, and in the $q \rightarrow 0$ limit uncertainties in the response functions grow. We conclude that physical observables are particularly sensitive to the large uncertainties we have found in the $q \rightarrow 0$ limit of some of the nuclear response functions in Eq. (3). This is one of the key results of the present analysis.

Figure 6 shows the differential rate of nuclear recoil events as a function of $\cos \theta$ for a ${ }^{4} \mathrm{He}$ detector and the interaction operators $\hat{\mathcal{O}}_{5}$ and $\hat{\mathcal{O}}_{15}$. We do not consider the interaction operator $\hat{\mathcal{O}}_{4}$, since the nuclear response functions $W_{\Sigma^{\prime}}^{\tau \tau^{\prime}}$ and $W_{\Sigma^{\prime \prime}}^{\tau \tau^{\prime}}$ are identically zero for ${ }^{4} \mathrm{He}$. Dark matter particle mass and coupling constants are set as above. As in the case of ${ }^{3} \mathrm{He}$, we find that nuclear physics uncertainties are large for $\hat{\mathcal{O}}_{15}$, which generates the $W_{\Phi^{\prime \prime}}^{\tau \tau^{\prime}}$ response. They are negligible for $\hat{\mathcal{O}}_{5}$, which generates the $W_{M}^{\tau \tau^{\prime}}$ response.

Figure 7 illustrates results analogous to those reported in Fig. 5, but now for the isovector component of the operators in the legend. For isovector dark matter-nucleon couplings,

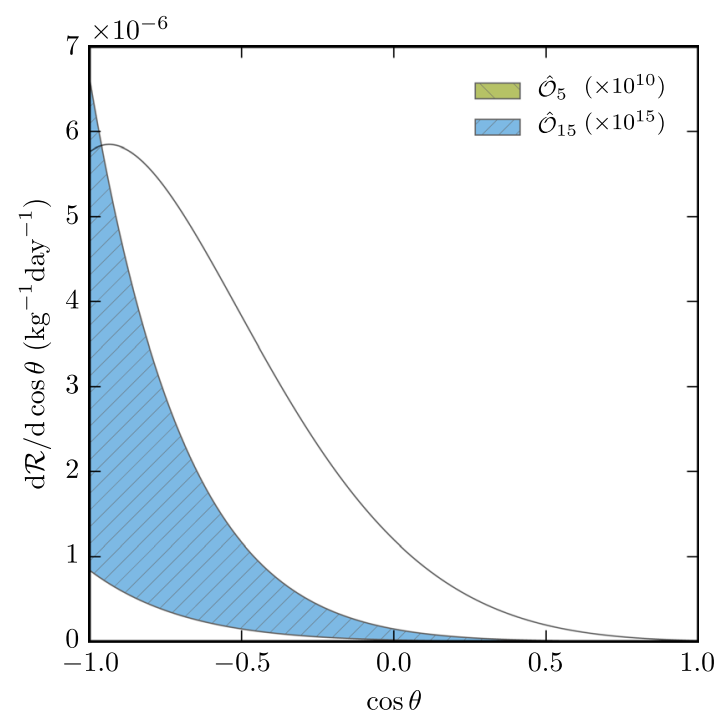

FIG. 6. Same as for Fig. 5, but now for a ${ }^{4} \mathrm{He}$ detector. Here we do not consider the interaction operator $\hat{\mathcal{O}}_{4}$ since the nuclear response functions $W_{\Sigma^{\prime}}^{\tau \tau^{\prime}}$ and $W_{\Sigma^{\prime \prime}}^{\tau \tau^{\prime}}$ are identically zero for ${ }^{4} \mathrm{He}$.

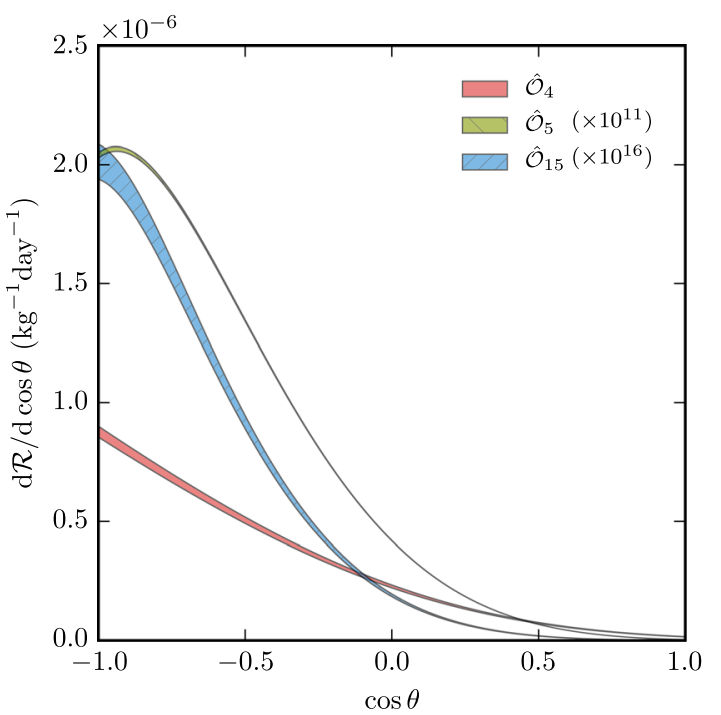

FIG. 7. Same as for Fig. 5, but for the isovector component of the operators in the legend.

nuclear physics uncertainties are only moderate. From the experimental perspective, we therefore conclude that this is the most favorable particle physics scenario.

In Fig. 8 we compare two independent calculations of the differential rate of nuclear recoil events, Eq. (20). The first calculation is the one we perform here in the $a b$ initio NCSM approach; the second one has been performed in

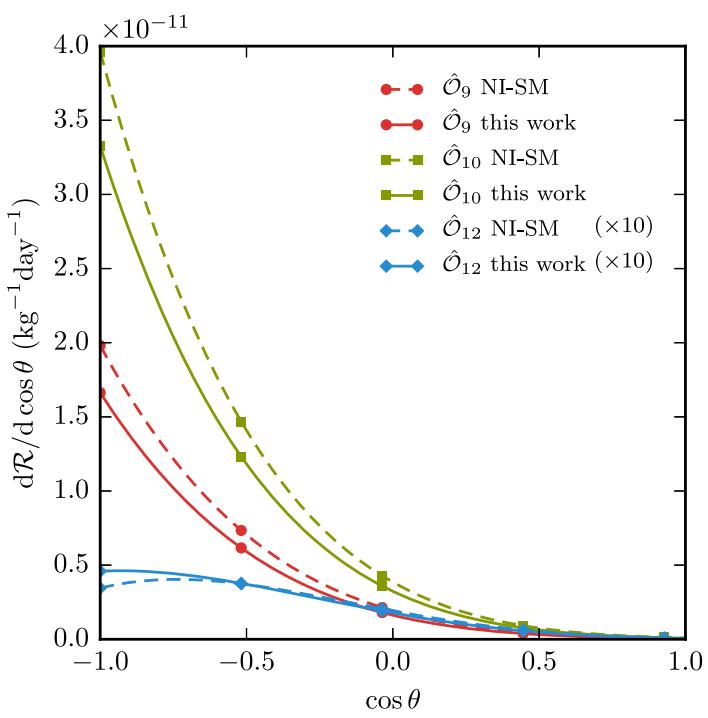

FIG. 8. Differential rate of nuclear recoil events computed within the $a b$ initio NCSM approach (this work) compared with the NI-SM rate found in [60]. We focus on the isoscalar component of the interaction operators listed in the legend and consider ${ }^{3} \mathrm{He}$ as a target material. We set $m_{\chi}=10 \mathrm{GeV}$ and the coupling constants to the reference value given in the text. The comparison is performed considering for each $a b$ initio nuclear response function the upper boundary of the corresponding nuclear physics uncertainty band found in Sec. III A. 
[60] using the nuclear SM technique. Results are presented for the isoscalar components of selected interaction operators, setting $m_{\chi}$ and associated coupling constants as above, and focusing on ${ }^{3} \mathrm{He}$ as a target material. The comparison is performed considering for each $a b$ initio nuclear response function the upper boundary of the corresponding nuclear physics uncertainty band found in Sec. III A. In Fig. 8 relative differences are moderate: $30 \%$ or less for all recoil directions. The ab initio calculation performed here predicts lower rates of recoil events for interaction operators which generate single nuclear response functions, such as the $\hat{\mathcal{O}}_{9}$ and $\hat{\mathcal{O}}_{10}$ operators. However, there are cases in which the ab initio calculation predicts three nuclear response functions different from zero, while the phenomenological approach predicts only two. This is the case of the operator $\hat{\mathcal{O}}_{12}$, for which ab initio and phenomenological calculation predict $W_{k}^{\tau \tau^{\prime}} \neq 0$ for $k=$ $\Sigma^{\prime}, \Sigma^{\prime \prime}, \Phi^{\prime \prime}$ and zero otherwise, and $W_{k}^{\tau \tau^{\prime}} \neq 0$ for $k=\Sigma^{\prime}, \Sigma^{\prime \prime}$ and zero otherwise, respectively. In these cases, ab initio rates are larger than phenomenological rates in a wide range of recoil directions.

\section{DISCUSSION AND OUTLOOK}

In this work we have developed an ab initio framework for computations of nuclear response functions for dark matter scattering off atomic nuclei. Our nuclear-structure calculations have been performed with the NCSM method, and applied to the study of light nuclei. However, our approach can be generalized to other ab initio methods and extended to heavier isotopes.

In particular we have quantified the uncertainties of nuclear response functions that result from the remaining freedom in the construction of realistic nuclear interactions. Furthermore, we have quantified the impact of such nuclear-physics uncertainties on physical observables that are relevant for dark matter searches. Particular emphasis has been placed on the rate of dark matter-nucleus scattering events at directional detection experiments. We have performed this calculation for a variegated set of dark matter-nucleon interactions. Depending on the type of nuclear response that is considered, relative uncertainties on the scattering rate can be as large as a factor of 5 for nuclear recoil directions antiparallel to the Earth's motion in the galactic rest frame (see Fig. 6). For comparison, current uncertainties on the local dark matter density are at the 30\% level [78], or smaller if knowledge of the baryonic mass density profile is assumed [73]. Uncertainties on the local dark matter velocity distribution can be significantly larger, but only affect results obtained for dark matter particle masses below $20 \mathrm{GeV}$ or so [74]. We have also compared scattering rates computed using the NI-SM with those from the $a b$ initio NCSM approach. For ${ }^{3,4} \mathrm{He}$ most differences are moderate or small, although with a clear dependence on the nuclear recoil direction. However, certain response functions that are evaluated to zero in the NI-SM approach can appear when allowing more freedom in the nuclear many-body model space. Consequently, we have identified scenarios in which the expected dark matter signal is larger when ab initio nuclearstructure input is used.

In this work we have used the NREFT description of the WIMP-nucleon interaction. However, it should be straightforward to implement also the alternative framework in which QCD constraints, imposed by chiral symmetry, are used to obtain the WIMP-nucleon interaction from an underlying interaction at the quark level. Such an extension would become relevant for the matching of parameters to new physics models, but also for an improved understanding of the relative importance of many-body currents in nuclei with larger mass numbers.

Further applications of the ab initio scheme that we have developed include improved calculations of (i) the rate of dark matter capture via scattering by nuclei in the Earth and in the Sun; (ii) the nuclear response functions for ${ }^{19} \mathrm{~F}$, which is used in the direct detection experiment PICO [79] and in directional detection experiments [42]; and (iii) the nuclear response functions for ${ }^{16} \mathrm{O}$, that is used in the direct detection experiment CRESST-II [80]. In order to maintain reasonable nuclear-physics uncertainties for predictions involving these heavier isotopes one might have to calibrate the chiral nuclear interaction differently from what has been done in this work. In particular, rather than staying exclusively in the few-body sector one might explore the alternative strategy of informing the nuclear-force model about low-energy many-body observables [81,82]. In addition, one could consider to include information on various electroweak observables, which would provide additional constraints on the relevant response functions.

Furthermore, medium-mass and heavier closed-shell nuclei can be within computational reach using nuclear structure methods that have a gentler scaling with the number of nucleons. In addition, such methods can be used to compute effective valence-space interactions for use in standard SM calculations [28,29]. A key point would be that such valence-space interactions will be constructed directly from the underlying $N N$ interaction using nonperturbative methods. With corresponding advances in SM technology, this approach opens the path towards $a b$ initio studies of nuclear responses for germanium and xenon isotopes with quantified uncertainties.

\section{ACKNOWLEDGMENTS}

This work has been supported by the Knut and Alice Wallenberg Foundation (PI: Jan Conrad) and is performed in the context of the Swedish Consortium for Direct Detection of Dark Matter (SweDCube). This research was also supported by the Munich Institute for Astroand Particle Physics (MIAPP) of the Deutsche Forschungsgemeinschaft (DFG) cluster of excellence 
"Origin and Structure of the Universe" and we thank the participants of the program on "Astro-, Particle and Nuclear Physics of Dark Matter Direct Detection" for many valuable discussions. Some of the computations were performed on resources provided by the Swedish National Infrastructure for Computing (SNIC) at National Supercomputer Centre in Sweden (NSC).

\section{APPENDIX: DARK MATTER RESPONSE FUNCTIONS}

Dark matter response functions introduced in Eq. (2) and used in Sec. III B:

$$
\begin{aligned}
R_{M}^{\tau \tau^{\tau^{\prime}}}\left(v_{q}^{\perp 2}, \frac{q^{2}}{m_{N}^{2}}\right)= & c_{1}^{\tau} c_{1}^{\tau^{\prime}}+\frac{J_{\chi}\left(J_{\chi}+1\right)}{3}\left(\frac{q^{2}}{m_{N}^{2}} v_{q}^{\perp 2} c_{5}^{\tau} c_{5}^{\tau^{\prime}}\right. \\
& \left.+v_{q}^{\perp 2} c_{8}^{\tau} c_{8}^{\tau^{\prime}}+\frac{q^{2}}{m_{N}^{2}} c_{11}^{\tau} c_{11}^{\tau^{\prime}}\right), \\
R_{\Phi^{\prime \prime}}^{\tau \tau^{\prime}}\left(v_{q}^{\perp 2}, \frac{q^{2}}{m_{N}^{2}}\right)= & \frac{q^{2}}{4 m_{N}^{2}} c_{3}^{\tau} c_{3}^{\tau^{\prime}}+\frac{J_{\chi}\left(J_{\chi}+1\right)}{12} \\
& \times\left(c_{12}^{\tau}-\frac{q^{2}}{m_{N}^{2}} c_{15}^{\tau}\right)\left(c_{12}^{\tau^{\prime}}-\frac{q^{2}}{m_{N}^{2}} c_{15}^{\tau^{\prime}}\right), \\
R_{\Phi^{\prime \prime} M}^{\tau \tau^{\prime}}\left(v_{q}^{\perp 2}, \frac{q^{2}}{m_{N}^{2}}\right)= & c_{3}^{\tau} c_{1}^{\tau^{\prime}}+\frac{J_{\chi}\left(J_{\chi}+1\right)}{3}\left(c_{12}^{\tau}-\frac{q^{2}}{m_{N}^{2}} c_{15}^{\tau}\right) c_{11}^{\tau^{\prime}}, \\
R_{\tilde{\Phi}^{\prime}}^{\tau \tau^{\prime}}\left(v_{q}^{\perp 2}, \frac{q^{2}}{m_{N}^{2}}\right)= & \frac{J_{\chi}\left(J_{\chi}+1\right)}{12}\left(c_{12}^{\tau} c_{12}^{\tau^{\tau^{\prime}}}+\frac{q^{2}}{m_{N}^{2}} c_{13}^{\tau} c_{13}^{\tau^{\prime}}\right),
\end{aligned}
$$

$$
\begin{aligned}
R_{\Sigma^{\prime \prime}}^{\tau \tau^{\prime}}\left(v_{q}^{\perp 2}, \frac{q^{2}}{m_{N}^{2}}\right)= & \frac{q^{2}}{4 m_{N}^{2}} c_{10}^{\tau} c_{10}^{\tau^{\prime}}+\frac{J_{\chi}\left(J_{\chi}+1\right)}{12}\left[c_{4}^{\tau} c_{4}^{\tau^{\prime}}\right. \\
& +\frac{q^{2}}{m_{N}^{2}}\left(c_{4}^{\tau} c_{6}^{\tau^{\prime}}+c_{6}^{\tau} c_{4}^{\tau^{\prime}}\right)+\frac{q^{4}}{m_{N}^{4}} c_{6}^{\tau} c_{6}^{\tau^{\prime}} \\
& \left.+v_{q}^{\perp 2} c_{12}^{\tau} c_{12}^{\tau^{\prime}}+\frac{q^{2}}{m_{N}^{2}} v_{q}^{\perp 2} c_{13}^{\tau} c_{13}^{\tau^{\tau^{\prime}}}\right], \\
R_{\Sigma^{\prime}}^{\tau \tau^{\prime}}\left(v_{q}^{\perp 2}, \frac{q^{2}}{m_{N}^{2}}\right)= & \frac{1}{8}\left(\frac{q^{2}}{m_{N}^{2}} v_{q}^{\perp 2} c_{3}^{\tau} c_{3}^{\tau^{\prime}}+v_{q}^{\perp 2} c_{7}^{\tau} c_{7}^{\tau^{\prime}}\right) \\
& +\frac{J_{\chi}\left(J_{\chi}+1\right)}{12}\left[c_{4}^{\tau} c_{4}^{\tau^{\prime}}+\frac{q^{2}}{m_{N}^{2}} c_{9}^{\tau} c_{9}^{\tau^{\prime}}\right. \\
& +\frac{v_{q}^{\perp 2}}{2}\left(c_{12}^{\tau}-\frac{q^{2}}{m_{N}^{2}} c_{15}^{\tau}\right)\left(c_{12}^{\tau^{\prime}}-\frac{q^{2}}{m_{N}^{2}} c_{q}^{\perp 2} c_{14}^{\tau} c_{14}^{\tau^{\prime}}\right], \\
R_{\Delta}^{\tau \tau^{\prime}}\left(v_{q}^{\perp 2}, \frac{q^{2}}{m_{N}^{2}}\right)= & \frac{J_{\chi}\left(J_{\chi}+1\right)}{3}\left(\frac{q^{2}}{m_{N}^{2}} c_{5}^{\tau} c_{5}^{\tau^{\prime}}+c_{8}^{\tau} c_{8}^{\tau^{\prime}}\right), \\
R_{\Delta \Sigma^{\prime}}^{\tau \tau^{\prime}}\left(v_{q}^{\perp 2}, \frac{q^{2}}{m_{N}^{2}}\right)= & \frac{J_{\chi}\left(J_{\chi}+1\right)}{3}\left(c_{5}^{\tau} c_{4}^{\tau^{\prime}}-c_{8}^{\tau} c_{9}^{\tau^{\prime}}\right) .
\end{aligned}
$$

For definitiveness, we assume $J_{\chi}=1 / 2$, where $J_{\chi}$ is the dark matter particle spin.
[1] G. Bertone and D. Hooper, arXiv:1605.04909.

[2] G. Jungman, M. Kamionkowski, and K. Griest, Phys. Rep. 267, 195 (1996).

[3] L. Bergström, Rep. Prog. Phys. 63, 793 (2000).

[4] G. Bertone, D. Hooper, and J. Silk, Phys. Rep. 405, 279 (2005).

[5] C.S. Frenk and S. D. M. White, Ann. Phys. 524, 507 (2012).

[6] L. Baudis, Phys. Dark Univ. 1, 94 (2012).

[7] S. Chang, A. Pierce, and N. Weiner, J. Cosmol. Astropart. Phys. 01 (2010) 006.

[8] J. Fan, M. Reece, and L.-T. Wang, J. Cosmol. Astropart. Phys. 11 (2010) 042.

[9] A. L. Fitzpatrick, W. Haxton, E. Katz, N. Lubbers, and Y. Xu, J. Cosmol. Astropart. Phys. 02 (2013) 004.

[10] V. Cirigliano, M. L. Graesser, and G. Ovanesyan, J. High Energy Phys. 10 (2012) 025.

[11] J. Menéndez, D. Gazit, and A. Schwenk, Phys. Rev. D 86, 103511 (2012).

[12] P. Klos, J. Menéndez, D. Gazit, and A. Schwenk, Phys. Rev. D 88, 083516 (2013).

[13] P. Klos, J. Menéndez, D. Gazit, and A. Schwenk, Phys. Rev. D 89, 029901 (2014).
[14] M. Hoferichter, P. Klos, and A. Schwenk, Phys. Lett. B 746, 410 (2015).

[15] M. Hoferichter, P. Klos, J. Menéndez, and A. Schwenk, Phys. Rev. D 94, 063505 (2016).

[16] U. van Kolck, Phys. Rev. C 49, 2932 (1994).

[17] E. Epelbaum, H.-W. Hammer, and U.-G. Meissner, Rev. Mod. Phys. 81, 1773 (2009).

[18] R. Machleidt and D. R. Entem, Phys. Rep. 503, 1 (2011).

[19] S. R. Beane, S. D. Cohen, W. Detmold, H. W. Lin, and M. J. Savage, Phys. Rev. D 89, 074505 (2014).

[20] F. D'Eramo and M. Procura, J. High Energy Phys. 04 (2015) 054.

[21] M. Cirelli, E. Del Nobile, and P. Panci, J. Cosmol. Astropart. Phys. 10 (2013) 019.

[22] J. Engel, S. Pittel, and P. Vogel, Int. J. Mod. Phys. E 01, 1 (1992).

[23] P. Toivanen, M. Kortelainen, J. Suhonen, and J. Toivanen, Phys. Rev. C 79, 044302 (2009).

[24] N. Anand, A. L. Fitzpatrick, and W. Haxton, Phys. Rev. C 89, 065501 (2014).

[25] R. Catena and B. Schwabe, J. Cosmol. Astropart. Phys. 04 (2015) 042. 
[26] B. Brown, Prog. Part. Nucl. Phys. 47, 517 (2001).

[27] E. Caurier, G. Martínez-Pinedo, F. Nowacki, A. Poves, and A. P. Zuker, Rev. Mod. Phys. 77, 427 (2005).

[28] G. R. Jansen, J. Engel, G. Hagen, P. Navratil, and A. Signoracci, Phys. Rev. Lett. 113, 142502 (2014).

[29] S. K. Bogner, H. Hergert, J. D. Holt, A. Schwenk, S. Binder, A. Calci, J. Langhammer, and R. Roth, Phys. Rev. Lett. 113, 142501 (2014).

[30] E. Dikmen, A. F. Lisetski, B. R. Barrett, P. Maris, A. M. Shirokov, and J. P. Vary, Phys. Rev. C 91, 064301 (2015).

[31] B. R. Barrett, P. Navrátil, and J. P. Vary, Prog. Part. Nucl. Phys. 69, 131 (2013).

[32] G. Hagen, T. Papenbrock, M. Hjorth-Jensen, and D. J. Dean, Rep. Prog. Phys. 77, 096302 (2014).

[33] J. Carlson, S. Gandolfi, F. Pederiva, S. C. Pieper, R. Schiavilla, K. E. Schmidt, and R. B. Wiringa, Rev. Mod. Phys. 87, 1067 (2015).

[34] H. Hergert, S. K. Bogner, T. D. Morris, A. Schwenk, and K. Tsukiyama, Phys. Rep. 621, 165 (2016).

[35] D. Lee, arXiv:1609.00421.

[36] C. Barbieri and A. Carbone, arXiv:1611.03923.

[37] E. Epelbaum, H. Krebs, and U.-G. Meißner, Eur. Phys. J. A 51, 53 (2015).

[38] R. J. Furnstahl, N. Klco, D. R. Phillips, and S. Wesolowski, Phys. Rev. C 92, 024005 (2015).

[39] B. D. Carlsson, A. Ekström, C. Forssén, D. F. Strömberg, G. R. Jansen, O. Lilja, M. Lindby, B. A. Mattsson, and K. A. Wendt, Phys. Rev. X 6, 011019 (2016).

[40] S. Binder et al., Phys. Rev. C 93, 044002 (2016).

[41] S. Ahlen et al., Int. J. Mod. Phys. A 25, 1 (2010).

[42] F. Mayet et al., Phys. Rep. 627, 1 (2016).

[43] E. Moulin, F. Naraghi, D. Santos, E. Collin, C. Winkelmann, Y. Bunkov, and H. Godfrin, arXiv:astro-ph/0309325.

[44] E. Moulin and D. Santos, arXiv:astro-ph/0505458.

[45] T. Franarin and M. Fairbairn, Phys. Rev. D 94, 053004 (2016).

[46] K. Schutz and K. M. Zurek, Phys. Rev. Lett. 117, 121302 (2016).

[47] J. B. Dent, L. M. Krauss, J. L. Newstead, and S. Sabharwal, Phys. Rev. D 92, 063515 (2015).

[48] A. L. Fitzpatrick, W. Haxton, E. Katz, N. Lubbers, and Y. Xu, arXiv:1211.2818.

[49] A. H. Peter, V. Gluscevic, A. M. Green, B. J. Kavanagh, and S. K. Lee, Phys. Dark Univ. 5-6, 45 (2014).

[50] R. J. Hill and M. P. Solon, Phys. Rev. Lett. 112, 211602 (2014).

[51] R. Catena and P. Gondolo, J. Cosmol. Astropart. Phys. 09 (2014) 045.

[52] R. Catena, J. Cosmol. Astropart. Phys. 09 (2014) 049.

[53] R. Catena, J. Cosmol. Astropart. Phys. 07 (2014) 055.

[54] V. Gluscevic and A. H. G. Peter, J. Cosmol. Astropart. Phys. 09 (2014) 040.

[55] P. Panci, Adv. High Energy Phys. 2014, 681312 (2014).

[56] L. Vietze, P. Klos, J. Menndez, W. C. Haxton, and A. Schwenk, Phys. Rev. D 91, 043520 (2015).
[57] G. Barello, S. Chang, and C. A. Newby, Phys. Rev. D 90, 094027 (2014).

[58] R. Catena and P. Gondolo, J. Cosmol. Astropart. Phys. 08 (2015) 022.

[59] K. Schneck et al. (SuperCDMS Collaboration), Phys. Rev. D 91, 092004 (2015).

[60] R. Catena, J. Cosmol. Astropart. Phys. 07 (2015) 026.

[61] B. J. Kavanagh, Phys. Rev. D 92, 023513 (2015).

[62] F. D'Eramo, B. J. Kavanagh, and P. Panci, J. High Energy Phys. 08 (2016) 111.

[63] R. Catena, A. Ibarra, and S. Wild, J. Cosmol. Astropart. Phys. 05 (2016) 039.

[64] F. Kahlhoefer and S. Wild, J. Cosmol. Astropart. Phys. 10 (2016) 032.

[65] G. Prézeau, A. Kurylov, M. Kamionkowski, and P. Vogel, Phys. Rev. Lett. 91, 231301 (2003).

[66] P. Navrátil, S. Quaglioni, I. Stetcu, and B. R. Barrett, J. Phys. G 36, 083101 (2009).

[67] P. Navrátil, G. P. Kamuntavičius, and B. R. Barrett, Phys. Rev. C 61, 044001 (2000).

[68] A. Ekström, G. Baardsen, C. Forssén, G. Hagen, M. HjorthJensen, G. R. Jansen, R. Machleidt, W. Nazarewicz, T. Papenbrock, J. Sarich, and S. M. Wild, Phys. Rev. Lett. 110, 192502 (2013).

[69] A. Ekström, B. D. Carlsson, K. A. Wendt, C. Forssén, M. Hjorth-Jensen, R. Machleidt, and S. M. Wild, J. Phys. G 42, 034003 (2015).

[70] R. Navarro Perez, J. E. Amaro, and E. R. Arriola, J. Phys. G 42, 034013 (2015).

[71] M. W. Goodman and E. Witten, Phys. Rev. D 31, 3059 (1985).

[72] D. N. Spergel, Phys. Rev. D 37, 1353 (1988).

[73] R. Catena and P. Ullio, J. Cosmol. Astropart. Phys. 08 (2010) 004.

[74] R. Catena and P. Ullio, J. Cosmol. Astropart. Phys. 05 (2012) 005.

[75] N. Bozorgnia, R. Catena, and T. Schwetz, J. Cosmol. Astropart. Phys. 12 (2013) 050.

[76] A. Crivellin, F. D’Eramo, and M. Procura, Phys. Rev. Lett. 112, 191304 (2014).

[77] F. D'Eramo, B. J. Kavanagh, and P. Panci, J. High Energy Phys. 08 (2016) 111.

[78] M. Pato, F. Iocco, and G. Bertone, J. Cosmol. Astropart. Phys. 12 (2015) 001.

[79] C. Amole et al. (PICO Collaboration), Phys. Rev. D 93, 061101 (2016).

[80] G. Angloher et al. (CRESST Collaboration), Eur. Phys. J. C 76, 25 (2016).

[81] A. Ekström, G. R. Jansen, K. A. Wendt, G. Hagen, T. Papenbrock, B. D. Carlsson, C. Forssén, M. Hjorth-Jensen, P. Navrátil, and W. Nazarewicz, Phys. Rev. C 91, 051301 (2015).

[82] G. Hagen et al., Nat. Phys. 12, 186 (2015). 